\title{
On detecting the large separation in the autocorrelation of stellar oscillation times series ${ }^{\star}$
}

\author{
B. Mosser $^{1}$ and T. Appourchaux ${ }^{2}$ \\ 1 LESIA, CNRS, Université Pierre et Marie Curie, Université Denis Diderot, Observatoire de Paris, 92195 Meudon Cedex, France \\ e-mail: benoit.mosser@obspm.fr \\ 2 Institut d'Astrophysique Spatiale, UMR8617, Université Paris XI, Bâtiment 121, 91405 Orsay Cedex, France
}

Received 21 July 2009 / Accepted 7 October 2009

\begin{abstract}
Context. The observations carried out by the space missions CoRoT and Kepler provide a large set of asteroseismic data. Their analysis requires an efficient procedure first to determine if a star reliably shows solar-like oscillations, second to measure the so-called large separation, third to estimate the asteroseismic information that can be retrieved from the Fourier spectrum.

Aims. In this paper we develop a procedure based on the autocorrelation of the seismic Fourier spectrum that is capable of providing measurements of the large and small frequency separations. The performance of the autocorrelation method needs to be assessed and quantified. We therefore searched for criteria able to predict the output that one can expect from the analysis by autocorrelation of a seismic time series.

Methods. First, the autocorrelation is properly scaled to take into account the contribution of white noise. Then we use the null hypothesis $H_{0}$ test to assess the reliability of the autocorrelation analysis. Calculations based on solar and CoRoT time series are performed to quantify the performance as a function of the amplitude of the autocorrelation signal.

Results. We obtain an empirical relation for the performance of the autocorrelation method. We show that the precision of the method increases with the observation length, and with the mean seismic amplitude-to-background ratio of the pressure modes to the power $1.5 \pm 0.05$. We propose an automated determination of the large separation, whose reliability is quantified by the $H_{0}$ test. We apply this method to analyze red giants observed by CoRoT. We estimate the expected performance for photometric time series of the Kepler mission. We demonstrate that the method makes it possible to distinguish $\ell=0$ from $\ell=1$ modes.

Conclusions. The envelope autocorrelation function (EACF) has proven to be very powerful for the determination of the large separation in noisy asteroseismic data, since it enables us to quantify the precision of the performance of different measurements: mean large separation, variation of the large separation with frequency, small separation and degree identification.
\end{abstract}

Key words. stars: oscillations - stars: interiors - methods: data analysis - methods: analytical

\section{Introduction}

Asteroseismology is known to be an efficient tool to analyze the stellar interior and to derive the physical laws that govern stellar structure and evolution. It benefits nowadays from highperformance photometric data provided by the space missions CoRoT (Baglin et al. 2006) and Kepler (Christensen-Dalsgaard et al. 2007). The amount of data is much higher than from the earlier ground-based observations, even with the recent multisite ground-based observations (Arentoft et al. 2008), since space-borne instruments are able to simultaneously record long time series on numerous targets. The data analysis then must be efficient enough to rapidly extract seismic information from hundreds to thousands of stars.

This task is principally carried out on the frequency pattern of the eigenmodes propagating inside the stars. For targets showing solar-like oscillations, this pattern follows the asymptotic relation of Tassoul (1980) providing eigenfrequencies nearly equally spaced by $\Delta v / 2$. The eigenfrequency of radial order $n$ and degree $\ell$ expresses $v_{n, \ell} \simeq[n+\ell / 2+\varepsilon] \Delta v-\ell(\ell+1) D_{0}$, $\Delta v$ being called the large separation, $D_{0}$ giving a measure of the

* The CoRoT space mission, launched on 2006 December 27, was developed and is operated by the CNES, with participation of the Science Programs of ESA, ESA's RSSD, Austria, Belgium, Brazil, Germany and Spain. small separation, and $\varepsilon$ a constant term. The determination of the large separation $\Delta v$ is the first step of any seismic analysis. If the signal-to-noise ratio is high enough, $\Delta v$ can be detected by eye in the power spectrum. In many cases, this is not possible, and the determination of $\Delta v$ requires sophisticated tools, as was the case for the first correct determination of the large separation of Procyon (Mosser et al. 1998) and of the first CoRoT target observed with Doppler measurements (Mosser et al. 2005). For observations dealing with a single target, the tools used for the determination of $\Delta v$ are usually unautomated and involve parameters specific to the target. Most often, they require the visual inspection of an image or a graph obtained by transforming the Fourier spectrum using the asymptotic relation cited above (échelle diagram or comb response). This step can be automated, but with great care, since the higher order terms of Tassoul (1980) complicate the stacking, as does, for example, the variation of the large separation reported in many asteroseismic targets (e.g. Mosser et al. 2008).

With the advent of space photometric missions, the use of pipelines for the automatic detection of $\Delta v$ is becoming mandatory (Mathur et al. 2009) since many targets have a low signal-tonoise ratio. A test to determine if the large separation is reliably detected is highly desirable, and a way to estimate the asteroseismic content of a high-precision photometric time series will be very helpful. 
This paper proposes an original way to address these issues. It is based on a first report by Roxburgh \& Vorontsov (2006, hereafter RV06), who analyse solar-like oscillations via the square of the autocorrelation of the time series, calculated as the Fourier spectrum of the filtered Fourier spectrum. RV06 state that the method is useful when faced with low signal-tonoise ratio data, and might be useful in obtaining information about a star even when individual frequencies cannot be extracted. Roxburgh (2009, hereafter R09) shows that it is possible, with basic and rapid computations, to attain more complex objectives, such as measurement of the variations of the large separation with frequency.

Since it provides a rapid measurement of the large separation, the autocorrelation method fits perfectly with the main asteroseismic objective of the Kepler mission, the large separation being used as an independent measurement in extracting the radius of stars hosting exoplanets, as in Stello et al. (2009). The autocorrelation achieves this goal without fitting a complex mode pattern to the stellar power spectrum. Therefore, it provides a simple tool to estimate the asteroseismic information of a Fourier spectrum or to use with Kepler, which will produce numerous time series of stellar targets.

We propose to quantify the relevance of the autocorrelation method with the null hypothesis, and to determine simple criteria to assess its efficiency and predictive power when analyzing an oscillation spectrum with a low signal-to-noise ratio. The method is also useful for extrapolating the performance obtained with a short time series to that obtained with a 4-year long time series, as will be provided by the Kepler mission. The analysis relies on photometric time series as observed by CoRoT (Baglin et al. 2006), plus simulations based on these CoRoT spectra with the addition of noise. It also includes simulations derived from a solar oscillation spectrum observed in photometry by the VIRGO/SPM instrument of the SOHO mission.

Section 2 introduces the envelope autocorrelation function (EACF) and the way we scale it to properly account for the noise contribution. We show in Sect. 3 how the value of the main autocorrelation peak varies with different global parameters of the stellar oscillation spectrum. A crucial parameter is the mean seismic height-to-background ratio $\mathcal{R}$, representing the smoothed height of the seismic power spectral density compared to the background. We introduce in Sect. 4 the $H_{0}$ test, that allows us to examine and to quantify the performance of the method. The value of the EACF gives a reliable criterion to estimate the seismic output, from the determination of the mean large separation when the signal is poor to the possibility of precise mode fitting in other cases. Discussion of various cases is presented in Sect. 5. We propose an automated determination of the large separation; using the $H_{0}$ test, we can quantify the reliability of this method. Section 6 is devoted to conclusions.

\section{Autocorrelation}

\subsection{Calculation}

RV06 proposes to perform the autocorrelation of the seismic time series as the Fourier spectrum of the filtered Fourier transform of the time series. This directly gives the amplitude of the envelope of the autocorrelation function, as shown in the Appendix. Instead of the canonical form,

$C(\tau)=\int x(t) x(t+\tau) \mathrm{d} t=\int X(v) X^{*}(v) \mathrm{e}^{\mathrm{i} 2 \pi v \tau} \mathrm{d} v$ with $X(v)$ the Fourier transform of $x(t)$, the autocorrelation with a filter $\mathcal{F}$ of width $\delta v_{\mathrm{H}}$ centered on $v_{\mathrm{c}}$ can be written:

$C=\int_{v_{\mathrm{c}}-\delta \nu_{\mathrm{H}}}^{v_{\mathrm{c}}+\delta v_{\mathrm{H}}} X(v) X^{*}(v) \mathcal{F}(v) \mathrm{e}^{\mathrm{i} 2 \pi v \tau} \mathrm{d} v$.

We deal with the dimensionless square module of the autocorrelation:

$\mathcal{A}^{\star}=\left|C(\tau)^{2}\right| /\left|C(0)^{2}\right|$.

The choice of square module has no impact on the results presented below, but proved to be more convenient in many cases, such as the observed linear increase of $\mathcal{A}^{\star}$ with the observing time (see Eq. (8)).

\subsection{Noise scaling}

In order to compare different cases, it is preferable to express the amplitude of the autocorrelation signal in noise units. The mean noise level in the autocorrelation can be derived from the fact that the noise statistic is a $\chi^{2}$ with 2 degrees of freedom. It is expressed in the general case as:

$\sigma=\frac{2}{N_{\mathrm{t}}} \frac{\left\langle\mathcal{F}^{2}\right\rangle}{\langle\mathcal{F}\rangle^{2}}$

with $N_{\mathrm{t}}$ the number of points in the time series. The noise level $\sigma$ is inversely proportional to the number $N_{\mathrm{H}}$ of frequency bins selected in the filter, when $N_{\mathrm{H}}$ is measured in a Fourier spectrum at the exact frequency resolution $\delta v=1 / T, T$ being the length of the observation, without oversampling. For a cosine filter (or Hanning function) of full-width at half-maximum $\delta v_{\mathrm{H}}$ centered on $v_{\mathrm{c}}$, one gets:

$\sigma_{\mathrm{H}}=\frac{3}{2 N_{\mathrm{H}}}$

With such a cosine filter and the resulting noise level, we define the EACF:

$\mathcal{A}=\mathcal{A}^{\star} / \sigma_{\mathrm{H}}$.

We note that $\mathcal{A}_{\Delta v}=\mathcal{A}\left(\tau_{\Delta v}\right)$, the amplitude of the first peak in the autocorrelation function, at a time shift $\tau_{\Delta v}=2 / \Delta v$. The first peak of the autocorrelation (Fig. A.1) is the signature of the autocorrelation of a seismic wavepacket after crossing the stellar diameter twice. As shon in RV06, measuring the time delay $\tau_{\Delta v}$ of this peak allows us to measure the large separation.

\section{Analysis}

We tested the variation of $\mathcal{A}_{\Delta v}$ with various parameters, in order to determine the relevant ingredients contributing to this signal. We based the analysis on solar data obtained with the VIRGO/SPM instrument onboard SOHO (Frohlich et al. 1997), and on the CoRoT data provided on the solar-like targets HD 49933 (Appourchaux et al. 2008), HD 49385 (Deheuvels et al., private comm.), HD 175726 (Mosser et al. 2009), HD 181420 (Barban et al. 2009) and HD 181906 (Garcia et al. 2009). We also include the red giant HD 181907 observed by CoRoT (Carrier et al. 2009). All these targets are presented in Table 1. We also considered a set of red giants observed in the exoplanetary field of CoRoT, already analyzed by Hekker et al. (2009). 
Table 1. $\mathcal{R}$ and parameters of the p mode envelope for CoRoT targets.

\begin{tabular}{|c|c|c|c|c|c|c|c|c|c|c|}
\hline$\overline{\text { Star }}$ & Type & $m_{\mathrm{V}}$ & $\begin{array}{r}{ }^{t} \\
\text { (day) }\end{array}$ & $\begin{array}{l}v_{\max } \\
\ldots \ldots(\mathrm{mH}\end{array}$ & $\begin{array}{l}\delta v_{\text {env }} \\
z) \ldots\end{array}$ & $\begin{array}{c}\langle\Delta v\rangle \\
(\mu \mathrm{Hz})\end{array}$ & $\alpha$ & $\gamma$ & $\mathcal{R}$ & $\mathcal{A}_{\max }$ \\
\hline HD $49385^{a}$ & G0IV & 7.41 & 136.9 & 1.00 & 0.54 & 56 & 0.95 & 9.7 & 0.90 & 452 \\
\hline HD $49933^{b 1}$ & F5V & 5.78 & 136.9 & 1.79 & 0.86 & 85 & 1.10 & 10.1 & 0.75 & 562 \\
\hline HD $49933^{b 2}$ & & & 60.7 & & & & & & & 237 \\
\hline HD $175726^{c}$ & G0V & 6.72 & 27.2 & 2.05 & 0.82 & 97 & 1.20 & 8.5 & 0.11 & 6.9 \\
\hline HD $181420^{d}$ & $\mathrm{~F} 2 \mathrm{~V}$ & 6.57 & 156.6 & 1.60 & 0.76 & 76 & 1.15 & 10.3 & 0.42 & 242 \\
\hline HD $181906^{e}$ & F8V & 7.6 & 156.6 & 1.92 & 0.88 & 85 & 1.00 & 10.3 & 0.15 & 47 \\
\hline HD $181907^{f}$ & G8III & 5.8 & 156.6 & 0.0286 & 0.0176 & 3.5 & 1.10 & 5.0 & 1.99 & 40 \\
\hline Sun/VIRGO ${ }^{g}$ & G2V & & 182.1 & 3.25 & 1.04 & 135 & 1.30 & 8.0 & 2.50 & $7.5 \times 10^{3}$ \\
\hline
\end{tabular}

$v_{\max }$ is the location of the frequency of maximum power; $\delta v_{\text {env }}$ is the full-width at half-maximum of the mode envelope; $\langle\Delta v\rangle$ is the mean value of the large separation; $\alpha$ represents the optimized filter width, in unit $\delta v_{\text {env }} ; \gamma$ represents $\delta v_{\text {env }}$ in unit $\Delta v ; \mathcal{R}$ measures the mean seismic amplitude in the time series compared to the noise, by the ratio in the Fourier spectrum, at the maximum-oscillation frequency, of the smoothed mode height to the background power density; $\mathcal{A}_{\max }$ measures the EACF.

References: ${ }^{a}$ Deheuvels et al. (private comm.); ${ }^{b 1}$ Benomar et al. (private comm.); ${ }^{b 2}$ Appourchaux et al. (2008); ${ }^{c}$ Mosser et al. (2009); ${ }^{d}$ Barban et al. (2009); ${ }^{e}$ Garcia et al. (2009); ${ }^{f}$ Carrier et al. (2009); ${ }^{g}$ Frohlich et al. (1997).

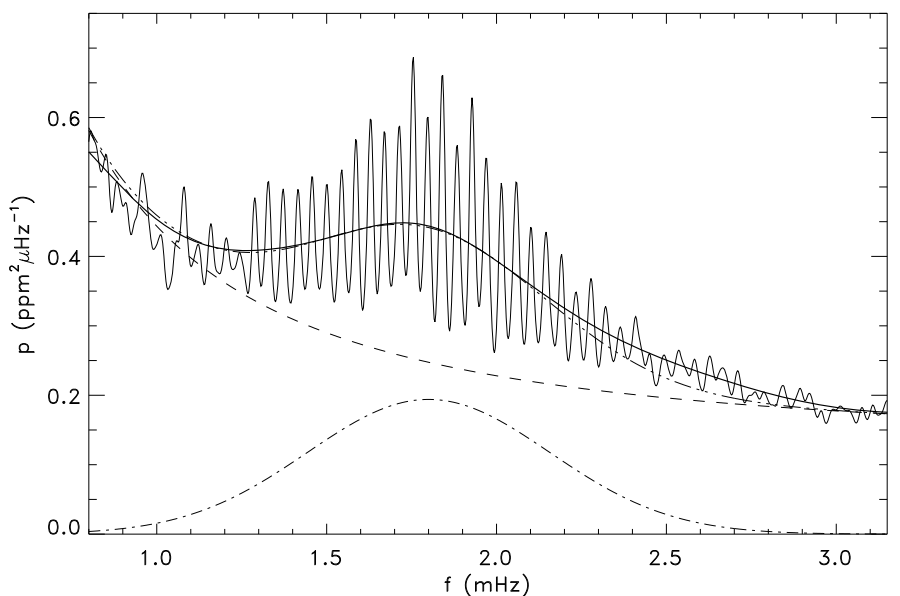

Fig. 1. Contributions to the smoothed power density distribution for HD 49933. The oscillation spectrum was slightly and severely smoothed (solid thin and thick lines). The dashed line represents the contributions of granulation and photon noise. The dash-dot lines account for the Gaussian modeling of the seismic envelope and the total contribution.

\subsection{Seismic amplitude-to-background ratio}

The strength of the autocorrelation of the time series depends on the ratio of the mean seismic amplitude compared to all other signal and noise. We can derive this signal-to-noise ratio in the time series from the ratio estimated in the oscillation spectrum. This ratio in the Fourier spectrum does not depend on the frequency resolution when the modes are resolved, i.e. the observation time is longer than the mode lifetime. In order to remove the influence of unknown parameters, such as the star inclination or the mode lifetime, we have to consider the ratio $\mathcal{R}$ of the mode height to the background power density, at the maximumoscillation frequency, in a smoothed power density spectrum (Fig. 1).

In order to estimate the background power, we have modeled the Fourier spectra with three components as in Michel et al. (2008): a low-frequency Lorentzian-like profile, a Gaussian mode envelope and a high-frequency noise. Figure 1 shows this modeling for HD 49933. The smoothed power density depends on the filter width. In order to avoid Gibbs phenomenon-like structures, a Gaussian filter has to be preferred to a boxcar average. The width has to be proportional to the large separation: a value of $3 \Delta v$ provides the optimum smoothing and limits the influence of the varying background level. Since, at this stage, the large separation is a priori unknown, the value of the filter width can be estimated with the help of the relation found between the large separation and the maximum-power frequency derived from the solar-like CoRoT targets:

$\Delta v \simeq(0.24 \pm 0.05) v_{\max }^{0.78 \pm 0.045} \quad$ (frequencies in $\mu \mathrm{Hz}$ ).

Table 1 gives $\mathcal{R}$ calculated for a set of CoRoT targets with solarlike oscillations, with the location $v_{\max }$ of the maximum PSD and the full-width at half-maximum $\delta v_{\text {env }}$ of the pressure mode envelope. The precision of the determination of $\mathcal{R}$ derived from these CoRoT targets is about $15 \%$.

\subsection{EACF as a function of time, filter width and signal-to-noise ratio}

The scaling (Eq. (6)) permitted us to perform different treatments in order to analyze how the EACF varies with the observing time $t$, the filter width $\delta v_{\mathrm{H}}$, the full-width at half-maximum of the mode envelope $\delta v_{\mathrm{env}}$ and $\mathcal{R}$. With a linear dependence of $t$ and the introduction of the reduced width $\mathcal{X}=\delta v_{\mathrm{H}} / \delta v_{\mathrm{env}}$, we found:

$\mathcal{A}_{\Delta v} \simeq 19.2 \mathcal{R}^{1.5}\left[\frac{t}{1 \text { day }}\right]\left[\frac{\delta v_{\mathrm{env}}}{1 \mathrm{mHz}}\right] \mathcal{X} \exp \left(-\frac{\mathcal{X}}{1.05}\right)$.

The amplitude $\mathcal{A}_{\Delta v}$ increases linearly with time, since $\sigma$ is inversely proportional to the observation time $t$ according to Eq. (5). As an important consequence, despite the limited lifetime of the modes, the precision of the seismic autocorrelation diagnosis increases linearly with $t$. This increase corresponds in fact to a decrease of $\sigma$ and therefore cannot saturate. The factor 1.05 in Eq. (8) is derived from the comparison between a Gaussian envelope and the Hanning filter: the best fit with such a filter requires a full width at half maximum equal to 1.05 times the one of the mode envelope.

Figure 2 shows the global fit, valid for photometric data of solar-like stars obtained with CoRoT or with VIRGO/SPM onboard SOHO. All values are fit within $\pm 20 \%$, when $\delta v_{\mathrm{H}} \leq$ $2 \delta v_{\text {env }}$, except the amplitudes for HD175726, which is the target with the lowest $\mathcal{R}$; however, the maximum amplitude for this star agrees with the others. 


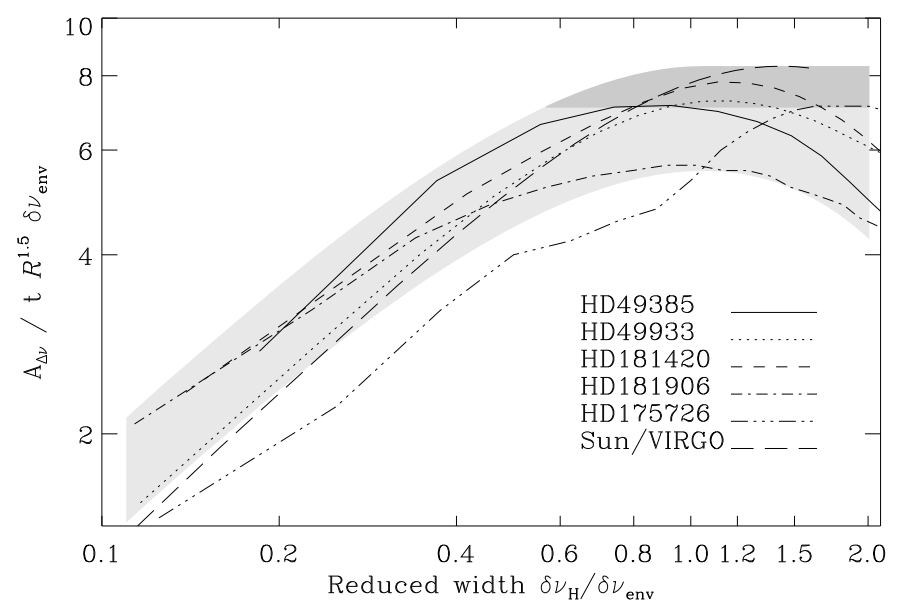

Fig. 2. Variation of the reduced amplitude $\mathcal{A}_{\Delta v} t^{-1} \mathcal{R}^{-1.5} \delta v_{\text {env }}^{-1}$ as a function of the reduced filter width $\delta v_{\mathrm{H}} / \delta v_{\text {env }}$. The light grey region indicates the validity of the global mean fit given by Eq. (8), within a $\pm 20 \%$ precision. The dark grey region indicates the location of the maxima, within $\mathrm{a} \pm 15 \%$ precision except for the double star HD 181906 .

We have verified that the exponent of the $\mathcal{R}$ dependence that minimizes the dispersion of the different curves in Fig. 2 is $1.5 \pm$ 0.05. A theoretical analysis should be performed to assess this result. Such a work requires one to take into account the link between $\mathcal{R}$ and the star inclination, the mode lifetime and the stellar noise.

\subsection{Maximum autocorrelation signal}

From Eq. (8), we can derive the maximum autocorrelation signal, obtained for $\delta v_{\mathrm{H}}=\alpha \delta v_{\mathrm{env}}$. It varies as:

$\mathcal{A}_{\text {max }} \simeq 7.0 \alpha \mathcal{R}^{1.5}\left[\frac{t}{1 \mathrm{day}}\right]\left[\frac{\delta v_{\mathrm{env}}}{1 \mathrm{mHz}}\right]$.

The parameter $\alpha$ is derived from the location of the maximum signal (Fig. 2). If $\alpha$ has not be determined, it should be replaced by its typical value $\alpha \simeq 1.05$ as used in Eq. (8).

Figure 2 helps to identify $\mathcal{A}_{\max }$. For all solar-like single stars but HD 181906, the agreement with Eq. (9) is better than $\pm 15 \%$. The fact that HD 181906 shows the lowest maximum among solar-like stars is certainly due to its binarity (Bruntt 2009): $\mathcal{R}$ and $\mathcal{A}_{\max }$ are corrupted by the unknown contribution of the companion. The observed value of $\mathcal{A}_{\max }$ for the red giant HD 181907, not shown, is 2 times lower than expected. This is clearly related to the narrow envelope of its oscillation spectrum, expressed by $\gamma=5$ compared to a mean value of 10 for solar-like stars (Table 1). The number of observed p modes is then twice as small and the EACF is reduced.

\section{Performance}

The scaling of $\mathcal{A}_{\max }$ with Eq. (6) allows us to test the reliability of the detection of the large separation with the $H_{0}$ test, and then to estimate the scientific output of the EACF. The null hypothesis, term first coined by the geneticist and statistician Ronald Fisher in Fisher (1935), consists here of assuming that the correlation is generated by pure white noise. If the EACF is high enough, the $H_{0}$ hypothesis is rejected, implying that a signal might have been detected (Appourchaux 2004).

\section{1. $H_{0}$ test}

Assessing the reliability of the measurement of the large separation as proposed by RV06 implies applying a statistical test as the null hypothesis $H_{0}$. A priori information on the large separation may come from scaling laws (Christensen-Dalsgaard \& Frandsen 1983), or may be derived from the location of the maximum signal, or from the initial guess of the stellar fundamental parameters. The large separation is then searched for over a range $\Delta \tau$. The number $\mathcal{N}$ of independent bins over the range $\Delta \tau$ depends on the width of the cosine filter. It is proportional but not equal to the number of points $N_{\mathrm{H}}$ selected by the filter in the Fourier spectrum. It can be determined from the full width at half-maximum $\delta \tau$ of the autocorrelation peaks. Then, $\mathcal{N}$ is:

$\mathcal{N}=\frac{\Delta \tau}{\delta \tau}$.

Therefore the rejection of the $H_{0}$ hypothesis at probability level $\mathcal{P}$ implies a threshold value:

$\mathcal{A}_{\lim } \simeq-\ln (\mathcal{P})+\ln \left(\frac{\Delta \tau}{\delta \tau}\right)$.

This equation is only valid if $\mathcal{P} \ll 1$. Equation (11) shows that the threshold increases with the searched range, but decreases with the resolution $\delta \tau$. We verified (see Eqs. (A.2) and (A.3)) that $\delta \tau$ is related to the width $\delta v_{\mathrm{H}}$ of the cosine filter. Then, $\mathcal{N}$ is

$\mathcal{N}=\frac{1}{\beta} \Delta \tau \delta v_{\mathrm{H}}$

with $\beta=0.763$. This number $\mathcal{N}$ can be estimated, even if nothing is known about the target, since $\Delta \tau$ and $\delta v_{\mathrm{H}}$ are both function of the large separation. The interval $\Delta \tau$ where the autocorrelation peak is to be found is measured by the time shift $\tau_{\Delta v}$. As a conservative value we may consider $\Delta \tau=\tau_{\Delta v}=2 / \Delta v$.

As shown above, the width of the best filter giving the maximum autocorrelation signal is proportional to the width of the seismic mode envelope, $\delta v_{\mathrm{H}}=\alpha \delta v_{\mathrm{env}}$, and the mode envelope also varies almost linearly with the the large separation, $\delta v_{\mathrm{env}}=\gamma \Delta v$. This gives $\delta v_{\mathrm{H}}=\alpha \gamma \Delta v$. As a consequence, independent of the large separation, the number of independent bins in the autocorrelation can be estimated by:

$\mathcal{N}=2 \frac{\alpha \gamma}{\beta}$.

Setting the mean optimum value of $\alpha$ to 1.05 and the mean value of $\gamma$ to 10, we obtain the number of independent bins to be considered in the EACF, about 28 . The threshold values for rejecting the $H_{0}$ hypothesis at level $\mathcal{P}=1 \%$ or $10 \%$ are then respectively 8.0 and 5.7 .

\subsection{Determination of the mean large separation $\langle\Delta v\rangle$}

The determination of the mean value of the large separation requires $\mathcal{A}_{\text {max }}$ to be greater than a threshold value of about 8 for a detection at the $1 \%$ rejection level. From Eq. (A.8), we then get an estimate of the relative precision of the mean large separation $\langle\Delta v\rangle$, integrated over a large frequency centered on the maximum-oscillation frequency:

$\frac{\delta\langle\Delta v\rangle}{\langle\Delta v\rangle} \simeq \frac{0.057}{\mathcal{A}_{\max }}$.

At the detection limit $\mathcal{A}_{\max }=8$, this gives a relative precision of about $0.6 \%$. The meaning of a mean value of the large separation is questionable, since most of the stars show significantly 


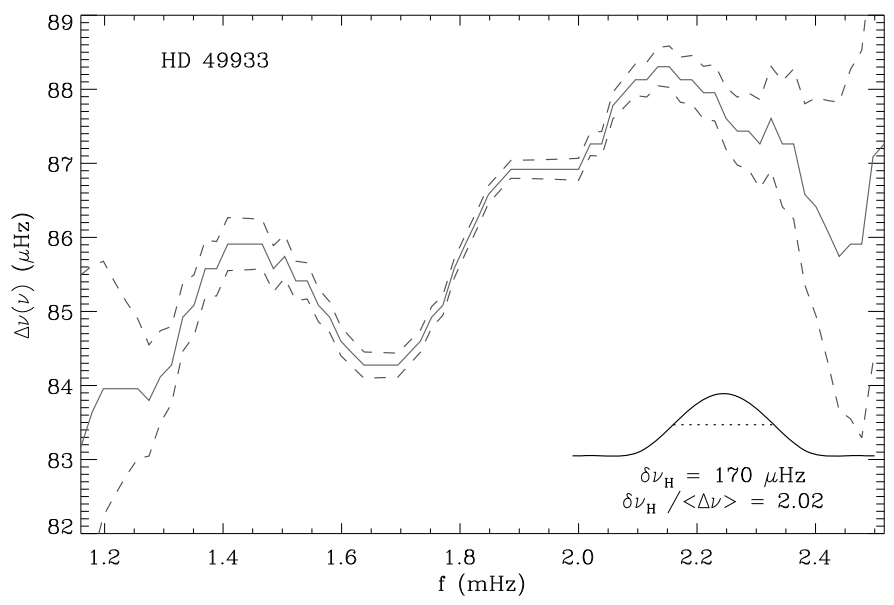

Fig. 3. Precision of the function $\Delta v(v)$ of HD 49933. 1- $\sigma$ error bars are given by the dashed lines. The inset shows the cosine filter, with a fullwidth at half-maximum equal to 2 times the mean large separation.

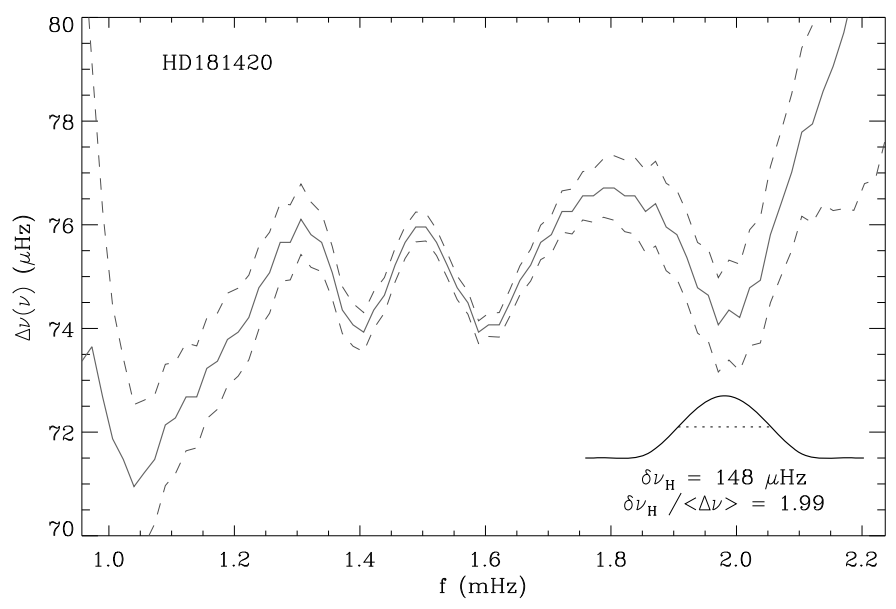

Fig. 4. Same as Fig. 3, but for HD 181420.

varying $\Delta v(v)$. These variations, integrated over the filter width, may limit the precision of $\langle\Delta v\rangle$, hence of an output value such as the stellar radius.

\subsection{Variation of the large separation with frequency}

With smaller values of $\delta v_{\mathrm{H}}$, it is possible to address the variation of the large separation with frequency as explained in R09. Investigating in detail $\Delta v(v)$ requires a filter $\delta v_{\mathrm{H}}$ much narrower than $\delta v_{\text {env }}$, so that we can derive $\mathcal{A} \simeq \mathcal{A}_{\max } e / \alpha \delta v_{\mathrm{H}} / \delta v_{\text {env }}$ from Eq. (8). In this case, the best relative precision at the maximum oscillation frequency can be derived from Eq. (A.8):

$\frac{\delta \Delta v}{\Delta v} \simeq \frac{0.6}{\mathcal{A}} \frac{\Delta v}{\delta v_{\mathrm{H}}} \simeq \frac{2.4}{\mathcal{A}_{\max }}\left(\frac{\delta v_{\mathrm{H}}}{\Delta v}\right)^{-2}$.

The scaling to the large separation insures a uniform precision throughout the HR diagram. With $\delta v_{\mathrm{H}} \simeq 2 \Delta v$, a $1 \%$ precision on the determination of $\Delta v(v)$ requires $\mathcal{A}_{\max } \geq 60$, which is achieved by most of the CoRoT targets (Table 1). Figures 3 and 4 show the precision we can obtain on the function $\Delta v(v)$.

\subsection{Disentangling the degree}

Examining the half-separations $\Delta_{01}=v_{n, 1}-v_{n, 0}$ and $\Delta_{10}=$ $v_{n+1,0}-v_{n, 1}$, as proposed by R09, requires $\delta v_{\mathrm{H}}$ narrower than

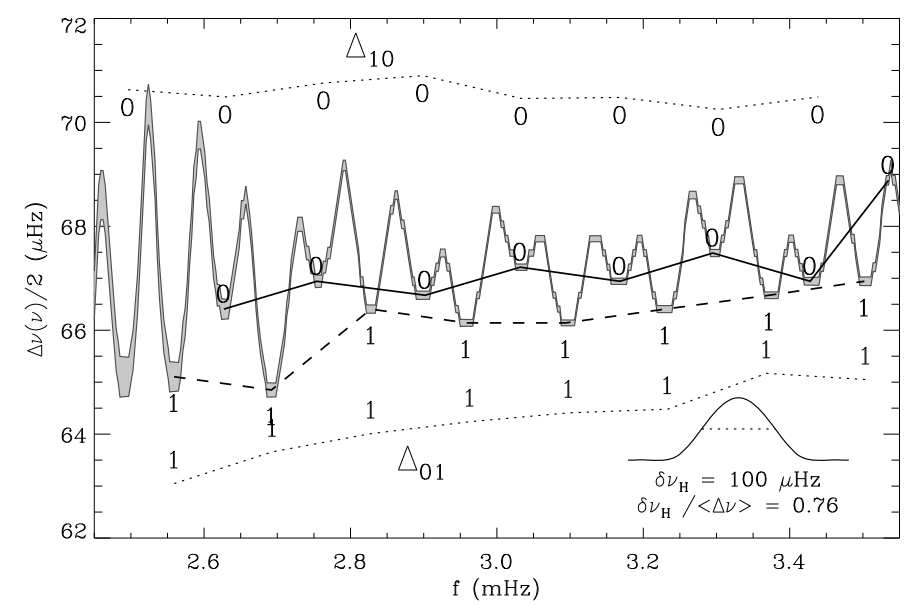

Fig. 5. Function $\Delta v(v)$ of the Sun, with a filter width equal to 0.75 times the mean large separation. The extrema of $\Delta v(v) / 2$ do not correspond to the curves $\Delta_{01}$ and $\Delta_{10}$, plotted as dotted curves. The symbols 0 and 1 indicate the location of the eigenfrequencies on the $\Delta_{01}$ and $\Delta_{10}$ curves, and indicate also the corresponding local minima of $\Delta v(v)$.

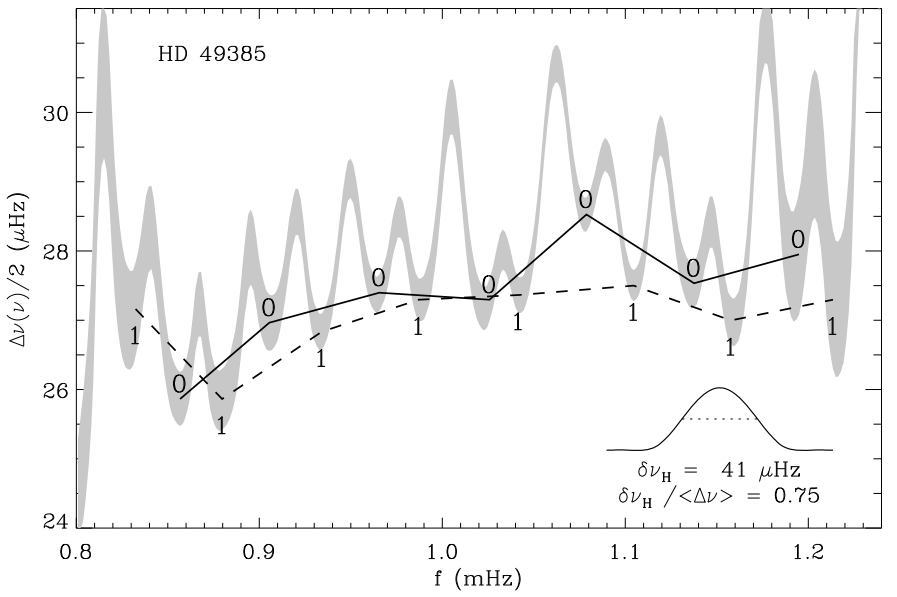

Fig. 6. Function $\Delta v(v)$ of $\mathrm{HD} 49385$, with a filter width equal to 0.75 times the mean large separation. The region in grey encompasses the values \pm the 1- $\sigma$ error bar. The local minima of $\Delta v(v)$, here marked by the degree, correspond to the eigenfrequencies mentioned by Deheuvels et al. (private comm.).

$\Delta v$. We have found that $\delta v_{\mathrm{H}} / \Delta v=0.75$ provides the best compromise: it is narrow enough to select only 1 pair of modes with degree 0 and 1, and large enough to give an accurate signal-toratio. Setting $\delta v_{\mathrm{H}} / \Delta v=0.75$, a $1 \%$ precision on the determination of $\Delta v$ requires $\mathcal{A}_{\max } \geq 430$, which is achieved only for HD 49933 and HD 49385.

As reported by R09, the different half-large separations are clearly distinguished. However, values are correlated within the filter, and mixed with other separations including $\ell=2$ modes. Therefore, we do not consider that the autocorrelation is able to provide a precise measurement of the half-separations. For instance, we cannot reproduce the Solar values (Fig. 5). However, we clearly show that the local minima match the eigenfrequencies with high precision. We observed, in the unambiguous cases provided by the Sun, HD 49385 (Fig. 6) and models, that the local minima associated with $\ell=1$ are lower than the ones with $\ell=0$. This is due to the fact that, under the assumption of a Tassoul-like spectrum, a narrow filter centered on the $\ell=1$ mode tests the separations $\Delta_{01}$ and $\Delta_{12}=v_{n, 2}-v_{n, 1}$, whereas a narrow filter centered on the $\ell=0$ mode tests the separations $\Delta_{10}$ 

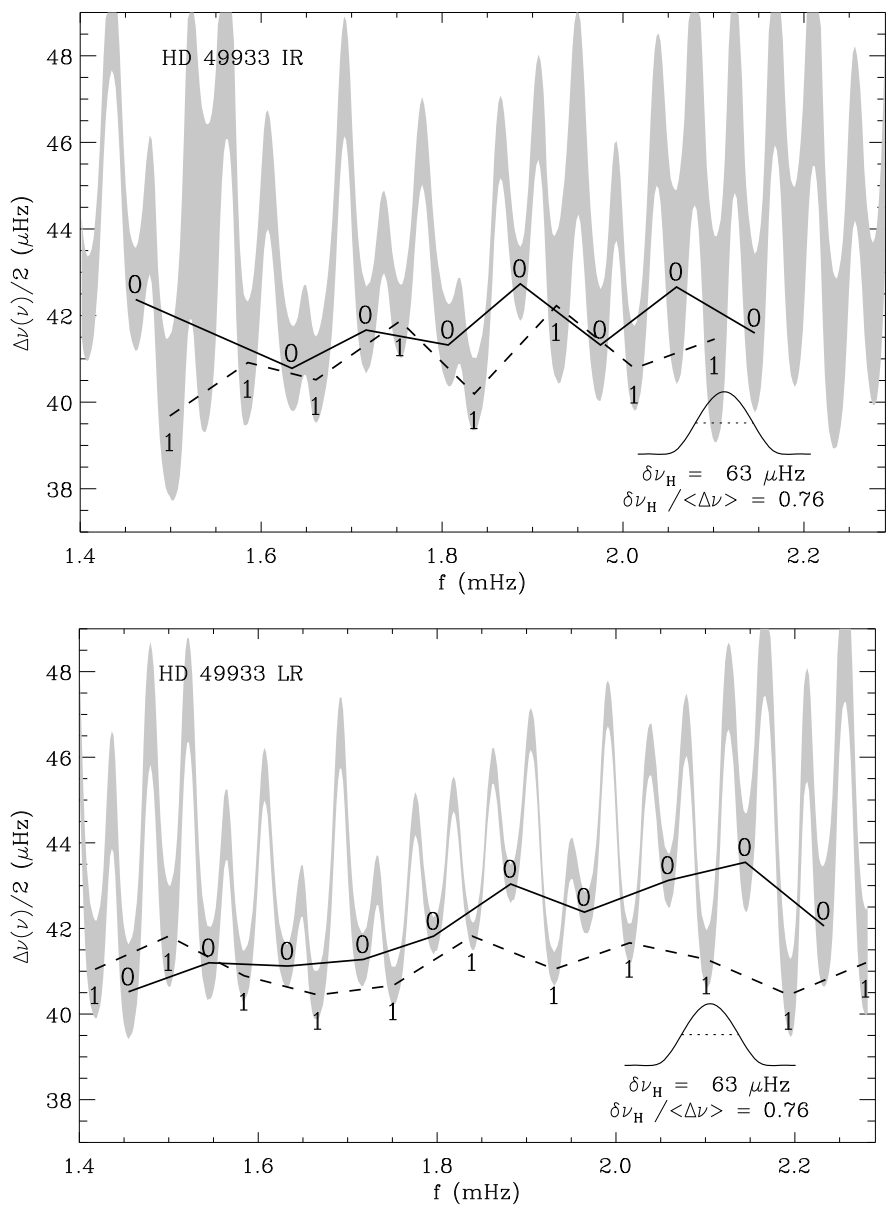

Fig. 7. Function $\Delta v(v)$ of HD 49933, with a filter width equal to 0.75 times the mean large separation. Radial and $\ell=1$ modes are identified. The two plots correspond to the 2 runs: the initial run IR lasted 60.7 days and the long run LR 136.9 days.

and $\Delta_{01}$. In the asymptotic formalism, $\Delta_{12}$ is significantly smaller than $\Delta_{10}$ (by an amount of $4 D_{0}$ ).

When the filter is not centered on an eigenmode, it mainly tests the separation $\Delta_{01}$ or $\Delta_{10}$. Therefore, we show that a narrow frequency windowed autocorrelation allows us to distinguish $\ell=0$ from $\ell=1$, which is a crucial issue since many observations have shown how difficult it can be to distinguish them (Barban et al. 2009; Garcia et al. 2009). The test applied to the first initial run on HD 49933 (Fig. 7) shows that the former mode identification of Appourchaux et al. (2008) cannot be confirmed, as also shown by Benomar et al. (private comm.) who analyze a second longer run.

A clear identification requires a signal-to-noise ratio high enough. Again, Eq. (15) allows us to estimate the autocorrelation amplitude required. In order to distinguish the small separation $D_{0}$, and considering as a rough estimate that in the mean case $D_{0}$ represents about $2 \%$ of the large separation, a reliable determination based on a narrow filter $\delta v_{\mathrm{H}}=0.75 \Delta v$ requires a maximum amplitude greater than about 200.

Table 2 summarizes the mean value of the difference $\delta_{01}$ between the local minima compared to the $1-\sigma$ uncertainty $\delta \Delta v / 2$ of the narrow frequency windowed autocorrelation function (Eq. (15)):

$\mathcal{T}_{01}=\frac{1}{\sqrt{\Delta n}} \sum_{i=1}^{\Delta n} \frac{\delta_{01 i}}{\delta \Delta v_{i}}$
Table 2. Degree identification.

\begin{tabular}{lrcc}
\hline \hline Star & $\Delta n$ & $\mathcal{T}_{01}$ & $p$-value (in\%) \\
\hline HD 49385 & 7 & 2.7 & 0.34 \\
HD 49933 IR & 8 & 2.0 & 2.22 \\
HD 49933 LR & 10 & 6.0 & 0.00 \\
HD 181420 & 6 & 3.1 & 0.10 \\
HD 181906 & 4 & 0.1 & 46.6 \\
\hline
\end{tabular}

IR and LR represent respectively the initial and long runs observation on HD 49933, from Appourchaux et al. (2008) and Benomar et al. (private comm.).

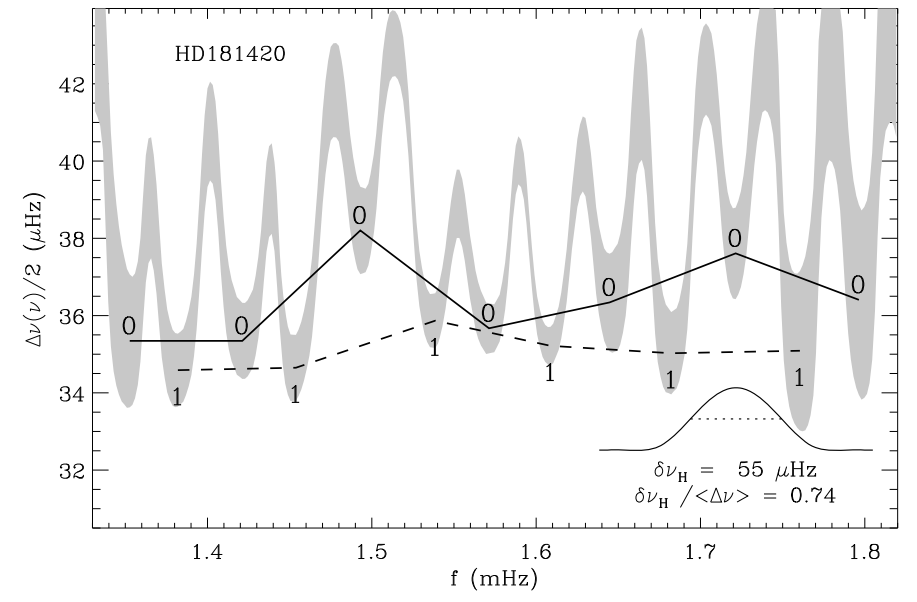

Fig. 8. Same as Fig. 7, but for HD 181420.

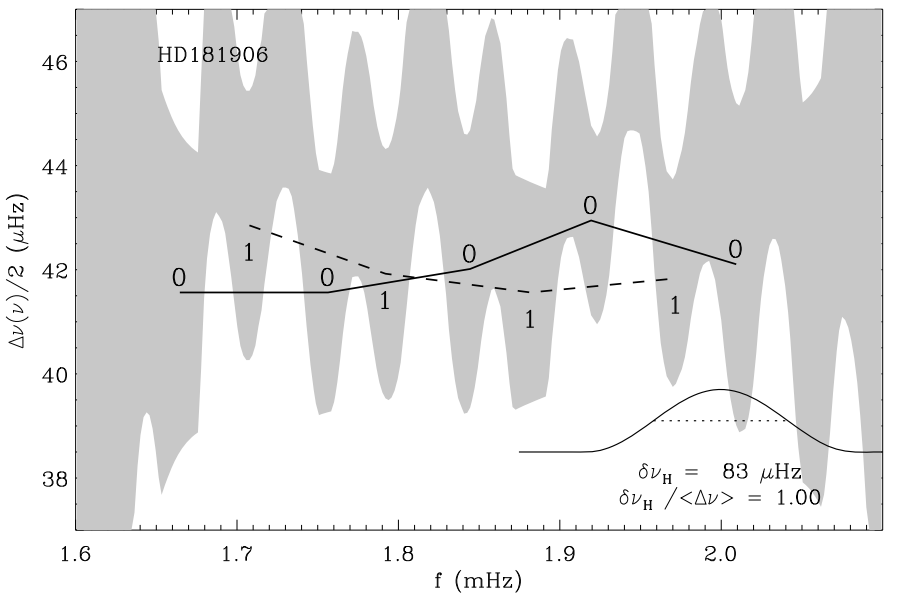

Fig. 9. Same as Fig. 7, but for HD 181906 and with a broader filter. The large uncertainty indicated by the broad grey region shows that the identification for that star is not reliable.

with $\Delta n$ the number of pairs of modes above the threshold level $\mathcal{A}=8$. Table 2 also provides the probability of obtaining a result as extreme as the observation assuming that the null hypothesis is true.

This criterion helps to explain why the ridges can be unambiguously identified in HD 49385 (Deheuvels et al., private comm.) and why scenario 1 for HD 181420 must be preferred (Barban et al. 2009): Figs. 7 and 8 show that the mean difference between the local minimal corresponding to $\ell=0$ or 1 is greater than the error bar of $\Delta v(v)$. On the other hand, no answer can be given for HD 181906 (Garcia et al. 2009): the low value of $\mathcal{A}_{\max }$ hampers the calculation of $\Delta v(v)$ with a narrow filter (Fig. 9). 
Table 3. Threshold levels.

\begin{aligned} & \hline \hline $\mathcal{A}_{\max }$ Detection \\ & \hline$<5.7$ no detection (10\% rejection level) \\ &$<8.0$ no detection (1\% rejection level) \\ & \hline 10 measurement of $\langle\Delta v\rangle$ with $0.5 \%$ precision \\ &$\geq 50$ fitting of the modes has proven to be possible \\ &$\geq 200$ identifying the mode degree has proven to be possible \\ &$\geq 300$ possible estimate of the small separation as in Roxburgh \\ & \& Vorontsov (2006) \\ & \hline\end{aligned}

The threshold values are given for a typical $\mathrm{F}$ dwarf. They can be made precise for a given star, according to its specific parameters $\alpha$ and $\gamma$. Levels are lower for red giants (to be estimated with $\gamma \simeq 4$ instead of 10).

The limited $\mathcal{A}_{\max } \simeq 250$ for the initial run on HD 49933 and the low value $\mathcal{T}_{01}$ help to explain the difficulties encountered with the mode identification given in Appourchaux et al. (2008).

\subsection{Ultimate precision on $\Delta v(v)$}

Obtaining the best time resolution in the EACF, namely the time resolution $\delta t$ of the time series, requires $\mathcal{A} \geq 1.21 / \delta v_{\mathrm{H}} \delta t$ for a filter width $\delta v_{\mathrm{H}}$ (cf. Eq. (A.10)). This relation imposes a strong constraint on the maximum amplitude. Furthermore, in order to investigate the variation $\Delta v(v)$, the condition has to be satisfied in a frequency range as large as $2 \delta v_{\mathrm{env}}$ around $v_{\mathrm{c}}$. This yields the condition:

$\mathcal{A} \geq \frac{4.9}{\delta v_{\mathrm{H}} \delta t}$.

According to this, high precision is easier to reach for high $\delta v_{\mathrm{H}}$ values, hence for low mass stars with a higher large separation. The decrease of the limit with increasing sampling $\delta t$ corresponds to a correlated decrease in resolution.

\subsection{Small separation}

Roxburgh \& Vorontsov (2006) proposed to make use of the autocorrelation function to obtain an independent estimate of the small separation. This method is based on the comparison of the peak amplitude of even or odd orders in the autocorrelation function. With $\mathcal{A}_{n}$ the amplitude of the peak of order $n$, it consists of comparing the decreasing $\mathcal{A}_{2 n}$ values to the increasing $\mathcal{A}_{2 n+1}$ (see Fig. 4 of RV06). Note that $\mathcal{A}_{\Delta v}$ corresponds to $\mathcal{A}_{2}$. Equality of the interpolated curves $\mathcal{A}_{2 n}$ and $\mathcal{A}_{2 n+1}$ occurs for $n$ of 3 or 4. Tests made on the available CoRoT data show that values of $\mathcal{A}_{2 n}$ larger than 15 are necessary to apply the method, therefore requiring very large values of $\mathcal{A}_{\Delta v}$, larger than about 300 . In the solar case, with the simulation including additional photon noise, the detection limit also occurs at $\mathcal{R} \simeq 300$.

\subsection{Threshold levels}

Table 3 summarizes the threshold levels for the determination of the seismic parameters with the EACF. At low signal-to-noise ratios, namely an $\mathcal{A}_{\text {max }}$ value lower than 8 , the method cannot operate, according to the $H_{0}$ test. Then, the domain where the autocorrelation is highly performing is for $\mathcal{A}_{\max }$ ranging from 8 (detection limit) to $\simeq 50$, when precise mode fitting becomes possible (HD 181906, Garcia et al. 2009). With $\mathcal{A}_{\max }$ value up

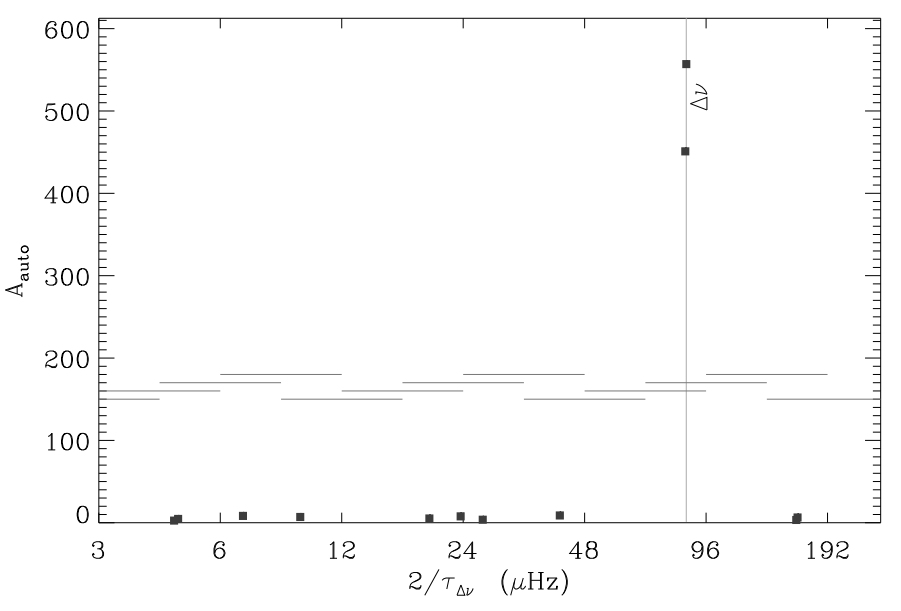

Fig. 10. Automatic search for the signature of a large separation for HD 49933. The grey line indicates the location of the maximum peak, corresponding to the mean large separation of the star. The horizontal segments indicate the ranges corresponding to the 13 initial guess values $\Delta v_{\mathrm{c}}$.

to 200, the EACF may be useful for identifying the degree of the modes, under the condition that the oscillation spectrum is close to a Tassoul-like pattern. Larger $\mathcal{A}_{\max }$ values allow a more detailed analysis with classical methods such as mode fitting (Appourchaux et al. 2006).

\section{Discussion}

\subsection{Automated determination of the large separation}

Autocorrelation may provide an effective automatic determination of the large separation when nothing is known about the star, as can be the case for a Kepler target. As shown previously, testing the autocorrelation around $\tau_{\Delta v}$ requires a cosine filter $\delta v_{\mathrm{H}}=\alpha \gamma \Delta v \simeq 10.5 \Delta v$, near the frequency $v_{\max }$. In order to perform the test in fully blind conditions, in a frequency range simultaneously encompassing giant and dwarf stars, $v_{\max }$ is derived from the scaling law given by Eq. (7).

The automatic test consists of analyzing the autocorrelation of the time series for a set of time shifts $\tau_{\Delta v_{\mathrm{c}}}$ in geometrical progression. We performed the automatic autocorrelation test with 13 values of $\Delta v_{\mathrm{c}}=2 / \tau_{\Delta v_{\mathrm{c}}}$, varying from 3 to $192 \mu \mathrm{Hz}$ with a geometric ratio $G$ equal to $\sqrt{2} ; \Delta v_{c}$ can be considered as an initial guess of the large separation. For each initial value $\Delta v_{\mathrm{c}}$, we explored the range $\left[\Delta v_{\mathrm{c}} / G, \Delta v_{\mathrm{c}} G\right]$ of the autocorrelation for 3 frequency ranges of the Fourier spectrum centered respectively around $v_{\max }$ and $v_{\max } \pm \delta v_{\mathrm{H}} / 2$. We finally derived the large separation from the maximum amplitude $\mathcal{A}_{\text {auto }}$ calculated for each $\Delta v_{\mathrm{c}}$ initial guess. Comparison of the different $\mathcal{A}_{\text {auto }}$ is made possible by the scaling provided by Eq. (6). Figure 10 shows the result for HD 49933.

We also tested the automatic test with the stars with the lowest $\mathcal{A}_{\max }$, namely HD175726 and HD 181907 . For HD 175726 , the single value exceeding the $10 \%$ rejection level occurs at $97 \mu \mathrm{Hz}$ (Fig. 11). This value of $\Delta v$ agrees with the solution proposed by Mosser et al. (2009). This detection is poor since a significance level of $10 \%$ means that the posterior probability of the null hypothesis is at least $38 \%$ according to Appourchaux et al. (2009). However, the automatic detection can be refined with a dedicated search with a more precise grid of analysis. In the case of HD 175726, the clear identification of an excess 


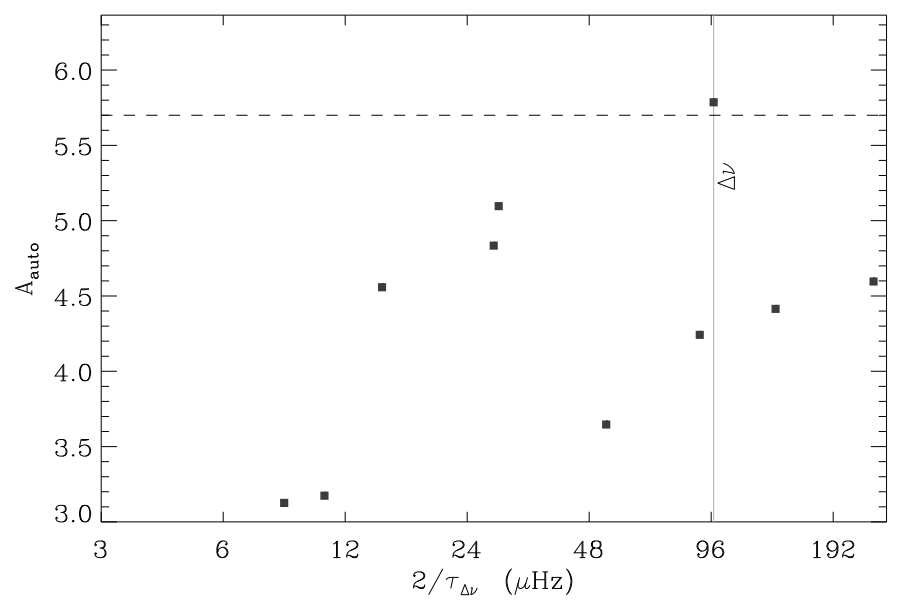

Fig. 11. Same as Fig. 10, but for HD 175726. The dashed line indicates the $10 \%$ rejection limit.

power centered at $2 \mathrm{mHz}$ first allows us to better estimate the parameters for searching $\Delta v$ and, second, gives a further indication that the measurement is reliable thanks to Eq. (7).

The amplitude $\mathcal{A}_{\text {auto }}$ of the automatic test is found to be close to the maximum amplitude $\mathcal{A}_{\text {max }}$. Only limited fine tuning around the automatically fixed parameters is needed to optimize the result. Mosser et al. (2009) have mentioned the difficulty of determining the large separation with other methods. The autocorrelation method proves to be powerful for a rapid estimate of the large separation; rapidity means here a few seconds of CPU time for the Fourier spectrum of the CoRoT time series, followed by a few seconds of CPU time for the automatic search with the autocorrelation, with a common laptop.

We verified that the method is effective for all other solar-like targets: it gives one single answer for $\Delta v$, and does not deliver any false positives. The case of red giants requires a dedicated analysis.

\subsection{Red giants}

We tested the method on the CoRoT red giant target HD 181907 (Carrier et al. 2009). With a large $\mathcal{R}$ for this target (about 2 ) and a large $\mathcal{A}_{\max }$ autocorrelation signal, the large separation is easily found around $3.5 \mu \mathrm{Hz}$, but the detection is polluted by many values clearly above the $1 \%$ rejection limit (Fig. 12). All these spurious detections are caused by artefacts: detection of the double of the large separation; detection of the diurnal frequency and its harmonics; detection of the CoRoT orbital frequency and half its value. We checked that the detections at high harmonics of the diurnal frequency are due to residuals of the window function (Mosser et al. 2009); they are introduced by the link between $v_{\max }$ and $\Delta v$ indicated by Eq. (7).

For HD 181907, Eq. (9) is valid within $30 \%$. As discussed in Sect. 3, the discrepancy compared to solar-like stars is due to the fact that the mode envelope of red giants is narrower than in solar-like stars.

The automated determination of the large frequency has been also tested on a set of 392 giants observed in the CoRoT field dedicated to exoplanetary science and analyzed by Hekker et al. (2009). The method proves to be efficient and rapid. It provides a clear advantage since it gives a quantified reliability thanks to the use of the $H_{0}$ test. We present in Fig. 13 the results obtained for these giants. The maximum oscillation frequency was calculated by Hekker et al. (2009). The amplitude of the

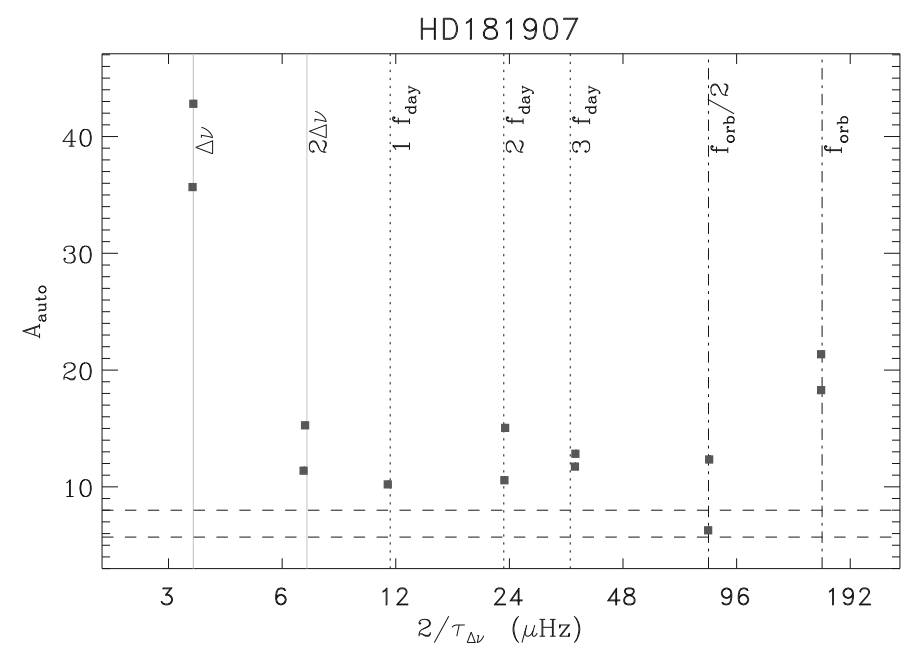

Fig. 12. Same as Fig. 11, but for the red giant HD 181907. The horizontal dashed lines indicate the $10 \%$ and $1 \%$ rejection limits. The vertical grey lines indicate the signature at $\Delta v$ and $2 \Delta v$, the dotted line the spurious signatures of the day aliases, and the dot-dashed lines the CoRoT orbital frequency and first subharmonic.

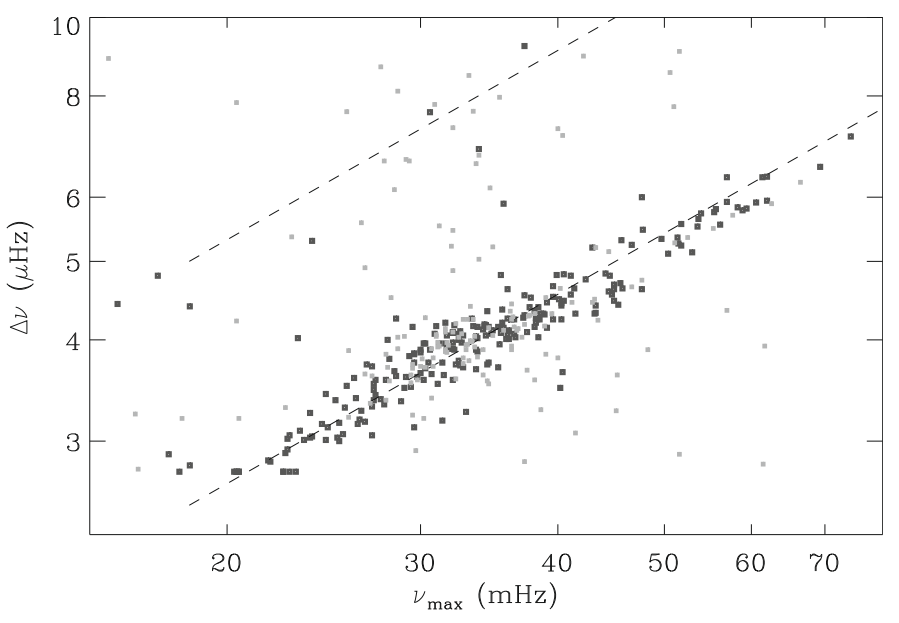

Fig. 13. Large separation, automatically measured for a set of 392 red giants analyzed in Hekker et al. (2009), as a function of the maximum oscillation frequency $v_{\max }$. Large black squares indicate positive detection; small grey squares correspond to unreliable cases. The dashed line indicate the global fit described by Eq. (18); a few cases correspond to the automatic detection of twice the large separation.

autocorrelation signal allows us to clearly discriminate artefacts from reliable detection ( $60 \%$ of the targets). The relative precision in the mean large separation is much better than $1 \%$, according to Eq. (14). After correction of the stars for which the double of the large separation is preferably automatically detected, we define from the fit of the relation between the large separation and the location of the maximum signal a power law varying as:

$\Delta v \simeq(0.26 \pm 0.015) v_{\max }^{0.78 \pm 0.03}$ (frequencies in $\mu \mathrm{Hz}$ ).

This law for giants is in agreement with Eq. (7) based on dwarfs and with Hekker et al. (2009).

\subsection{Kepler data}

The Kepler mission compared to CoRoT will provide different photometric performance, on dimmer targets but in some cases 
Table 4. Kepler performance.

\begin{tabular}{llrrrrrrrrrrr}
\hline \hline \multirow{2}{*}{$\begin{array}{l}\text { Stellar } \\
\text { type as }\end{array}$} & \multirow{2}{*}{ Type } & \multirow{2}{*}{$B-V$} & \multicolumn{4}{c}{ 90-day run } & \multicolumn{4}{c}{ 4-year run } \\
\hline HD 181907 & G8III & 1.09 & 23 & 23 & 23 & 23 & 22 & 379 & 378 & 377 & 373 & 364 \\
\hline HD 49385 & G0IV & 0.51 & 167 & 53 & 14 & 3.6 & 0.9 & 2709 & 864 & 233 & 58 & 14 \\
\hline HD 49933 & F5V & 0.35 & 44 & 11 & 2.9 & 0.7 & 0.2 & 715 & 188 & 46 & 11 & 2.7 \\
HD 181420 & F2V & 0.40 & 36 & 10 & 2.5 & 0.6 & 0.1 & 598 & 161 & 40 & 9.8 & 2.3 \\
HD 181906 & F8V & 0.43 & 27 & 6.9 & 1.7 & 0.4 & 0.1 & 446 & 111 & 27 & 6.5 & 1.5 \\
HD 175726 & G0V & 0.53 & 3.3 & 0.8 & 0.2 & 0.0 & 0.0 & 54 & 13 & 3.3 & 0.8 & 0.2 \\
\hline
\end{tabular}

Maximum amplitude $\mathcal{A}_{\max }$ for Kepler performance on CoRoT-like targets with varying magnitudes.

with longer observation duration (Christensen-Dalsgaard et al. 2007). According to Kepler performance (Kjeldsen et al. 2008), the noise level is about $0.92,10.2$ and $144 \mathrm{ppm}^{2} \mu \mathrm{Hz}^{-1}$ for targets of $V$ magnitude respectively equal to $9,11.5$ and 14 .

We can extrapolate the performance obtained with Kepler on targets similar to the ones observed by CoRoT, but of magnitude 9 to 14 , after a 4 -year long observation. Table 4 gives the amplitude $\mathcal{A}_{\max }$ for targets observed during typical 90-day or 4-year long runs. According to the expected performance in 90-day runs, the brightest F-type or the class IV targets will have a signal-to-noise ratio high enough to derive information on the large separation. In a 4-year run, the brightest $\mathrm{G}$ dwarfs will deliver a clean seismic signature. On the other hand, faint $F$ targets will have fully exploitable Fourier spectra that will require a precise mode fitting for the most complete seismic analysis. The performance for giants appears to be almost independent of the magnitude, since the contribution of photon noise is negligible at low frequency.

We can compare this approach to the hare-and-hounds exercises performed by Chaplin et al. (2008). The asteroseismic goal of Kepler is principally to derive information on stars hosting a planet, by the determination of the large separation. Compared to global fitting, the autocorrelation function gives a more rapid and direct answer.

The autocorrelation benefits from the rapid cadence (32 s) provided by CoRoT in the seismology field. Kepler will provide 2 cadences, at 1 or $30 \mathrm{~min}$. This yields a lower resolution in time, hence a lower precision on the expected results.

\section{Conclusion}

Roxburgh \& Vorontsov (2006) have proposed a method for estimating large and small separations from the analysis of the autocorrelation function. Roxburgh (2009) has extended the method to determine the variation of the large separation. In this paper, we have developed and quantified the method, relating the amplitude of the correlation peak at time shift $\tau_{\Delta v}=2 / \Delta v$ to various parameters.

We have scaled the autocorrelation to the white noise contribution, so that we were able to relate the autocorrelation signal to the mean seismic height-to-background ratio $\mathcal{R}$ that measures the relative power density of the signal compared to noise and to background signals. This empirical relation is precise to about $15 \%$ for solar-like stars. $\mathcal{R}$ aggregates the influence of unknown parameters such as the mode lifetimes, the star inclination (that governs the modes visibility) or the rotational splitting. On the other hand, all these unknown parameters complicate and slow down the fitting of individual eigenfrequencies. Therefore, the EACF shows here a possible advantage in terms of speed.
The EACF gives a direct measurement of the mean large separation. Compared to other methods, the estimate is accurate and simple, with an intrinsic threshold value, with error bars, and without any modeling of the other components of the Fourier spectrum (granulation or activity). Furthermore, when the signal-to-noise ratio is high enough, the EACF allows the measurement of the variation of the large separation with frequency, without any mode fitting. This is a key point for stellar radius measurement. Previous works have shown the difficulty to disentangle $\ell=0$ from $\ell=1$ modes in oscillation spectra of $F$ stars observed in photometry. We have verified that, for high signal-to-noise ratio Fourier spectra, the autocorrelation analysis can provide an unambiguous identification of the mode degree for a solar-like oscillation spectrum.

We have defined a method for the automatic determination of the large separation, which is efficient at low signal-to-noise ratio, even if no information is known for the star. We have determined that the width of the cosine filter used in the method that optimizes the EACF is very close to full-width at half-maximum of the mode envelope (ratio about 1.05). We have also checked that the performance of the method increases linearly with the duration of the time series. With very limited CPU time (a few seconds), this method delivers the mean large separation of a target. It requires no information on the star; it just relies on the assumption that the location of the excess power and its width are related to the large separation by a scaling law, what is verified for red giants and solar-like stars. Finally, we were able to investigate in a simple manner the capability of Kepler.

We are confident that the autocorrelation method will be of great help in analyzing high duty cycle time series as a complement to the Fourier analysis. As noticed by Fossat et al. (1999), the autocorrelation signal gives a clear signature since the autocorrelation delay, namely four times the stellar acoustic radius (about 4 to $8 \mathrm{~h}$ for an $\mathrm{F}$ dwarf), is much shorter than the mode lifetime (a few days). This allows each wavepacket to properly correlate with itself after a double travel along the stellar diameter, so that the autocorrelation integrates phased responses over the total duration time. In the Fourier spectrum, on the contrary, interference between the short-lived wavepackets observed in the time series produce a complicated pattern. But Fourier analysis still remains required for the precise determination of the eigenfrequencies derived from an accurate mode fitting.

\section{Appendix A: Performance of the autocorrelation}

\section{A.1. Square module of the autocorrelation}

The EACF presented in Sect. 2 is defined to directly give the envelope of the autocorrelation. Since negative frequencies are omitted, the EACF is related to the canonical autocorrelation $C_{ \pm}$, 


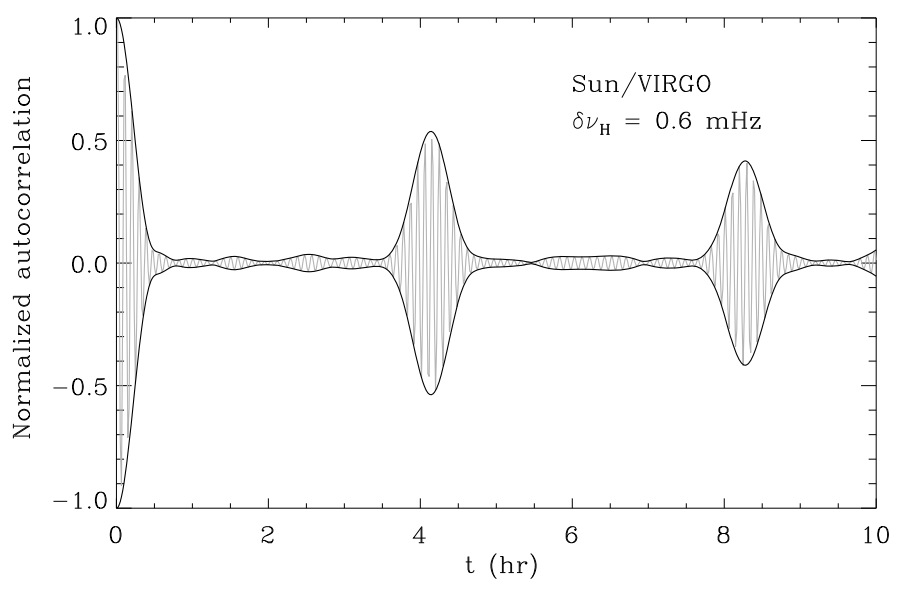

Fig. A.1. Comparison of $\pm|C|$ (black curves) and $C_{ \pm}$(grey line), both normalized to 1 at time shift 0 , calculated for the narrow frequency windowed solar Virgo spectrum.

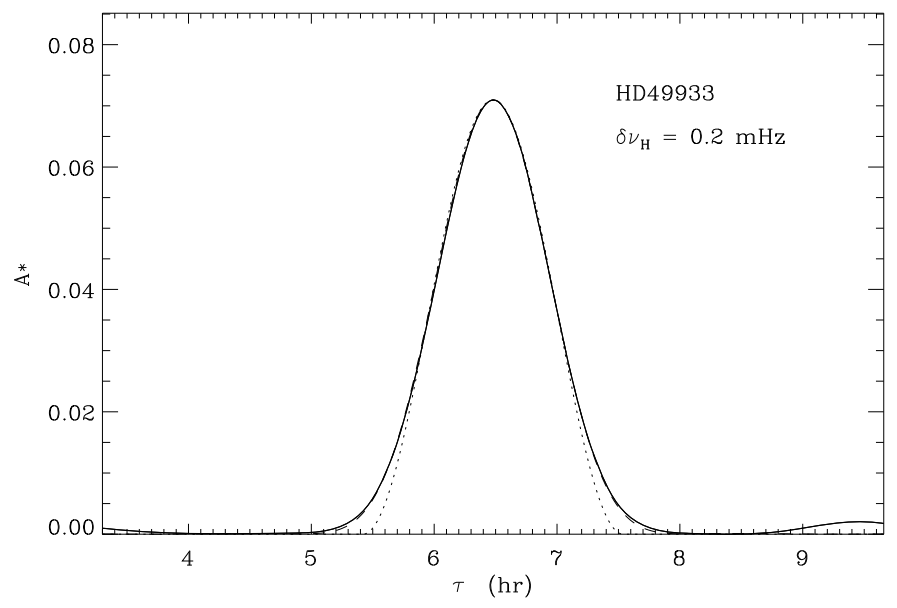

Fig. A.2. Autocorrelation peak $\mathcal{A}_{\Delta v}{ }^{\star}$ (solid line) normalized to 1 at time shift 0 , theoretical profile (dashed line) and cosine model (dotted line) calculated from the HD 49933 power spectrum and a filter width $\delta v_{\mathrm{H}}=$ $0.2 \mathrm{mHz}$.

that includes positive and negative frequencies of the Fourier spectrum, by:

$\mathcal{A} \propto|C|^{2}$ and $|C|=\sqrt{{C_{ \pm}{ }^{2}+\tilde{\mathcal{H}}\left(C_{ \pm}\right)^{2}}^{2}}$

where $\tilde{\mathcal{H}}$ is the Hilbert transform. Figure A.1 shows the difference between $C$ and $C_{ \pm}$, both functions include the contribution of a narrow Hanning filter.

\section{A.2. Autocorrelation peak}

The shape of the autocorrelation peaks is given by the Fourier transform of the Hanning filter, which can be expressed as the sum of 3 components (Max \& Lacoume 1996):

$\mathcal{H}(\tau) \propto 2 \operatorname{sinc}\left(\delta v_{\mathrm{H}} \tau\right)+\operatorname{sinc}\left(\delta v_{\mathrm{H}} \tau+1\right)+\operatorname{sinc}\left(\delta v_{\mathrm{H}} \tau-1\right)$

with $\operatorname{sinc} X=\sin (\pi X) / \pi X$. This global shape may be more simply modeled with a single cosine shape (Fig. A.2). In order to enhance the precision of the fit in the upper part of the autocorrelation peak, the full width at half maximum $\delta \tau$ of this cosine fit is:

$\delta \tau=\frac{\beta}{\delta v_{\mathrm{H}}}$ with $\beta \simeq 0.763$.
The flanks of the peak are not well fitted, which is unimportant compared to the fact that the fit above half-maximum performs well. $\delta \tau$ is much greater than the resolution time $\delta t$.

Precise determination of the large separation requires precise location of the peak maximum. In order to estimate the performance, we describe a peak as:

$$
\begin{cases}S(t)=\frac{A}{2}\left[1+\cos \pi \frac{t-\tau_{\Delta v}}{\delta \tau}\right] & \text { for }|t| \leq \delta \tau, \\ S(t)=0 & \text { for }|t|>\delta \tau .\end{cases}
$$

This fit shows variation:

$\mathrm{d} S_{\mathrm{A}}=-\frac{\pi A}{2 \delta \tau} \sin \pi \frac{t-\tau_{\Delta v}}{\delta \tau} \mathrm{d} t$

We can compare the variation of the signal peaking at amplitude $\mathcal{A}$ to the maximum variation of a noise contribution of amplitude $b$. At a time shift $\Delta t$ from the maximum, the signal variation and the maximum noise contribution are:

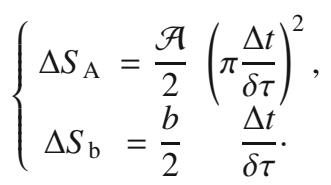

The precise identification of the signal maximum requires $\Delta S_{\mathrm{A}} \geq \Delta S_{\mathrm{b}}$, which translates into the condition:

$$
\pi \mathcal{A} \Delta t \geq b \delta \tau
$$

It is possible to interpret this condition as follows.

\section{A.3. Precision on the mean large separation}

If the amplitude is not large enough, then Eq. (A.7) defines a resolution $\Delta t$, hence a limited precision on the large separation:

$\frac{\delta \Delta v}{\Delta v}=\frac{\Delta t}{\tau_{\Delta v}}=\frac{\beta}{2 \pi} \frac{b}{\mathcal{A}} \frac{\Delta v}{\delta v_{\mathrm{H}}}$

We can set, as a limit to detection, $b=5$ and $\mathcal{A}=8$.

\section{A.4. Full resolution for the measurement of $\Delta v(v)$}

In order to recover the full time resolution $(\Delta t=\delta t)$, the amplitude must satisfy $\mathcal{A} \geq \mathcal{A}_{\delta t}$, with the definition:

$\mathcal{A}_{\delta t}=\frac{b}{\pi} \frac{\delta \tau}{\delta t}=\frac{b \beta}{\pi} \frac{1}{\delta v_{\mathrm{H}} \delta t}$.

With $b=5$ :

$\mathcal{A}_{\delta t} \simeq \frac{1.21}{\delta v_{\mathrm{H}} \delta t}$

Acknowledgements. This work was supported by the Centre National d'Etudes Spatiales (CNES). It is based on observations with CoRoT. Solar data were obtained from SOHO, a mission of international collaboration between ESA and NASA.

The work has benefitted from simulating discussions among asteroseismologists working on the excellent CoRoT data. B.M. thanks Ian Roxburgh for motivating discussions, and John Leibacher for helpful comments. We thank Saskia Hekker and Caroline Barban for providing us with the red giant data and the values of the maximum frequency plotted in Fig. 13. 


\section{References}

Appourchaux, T. 2004, A\&A, 428, 1039

Appourchaux, T., Berthomieu, G., Michel, E., et al. 2006, ESA SP, 1306, 377

Appourchaux, T., Michel, E., Auvergne, M., et al. 2008, A\&A, 488, 705

Appourchaux, T., Samadi, R., \& Dupret, M.-A. 2009, A\&A, 506, 1

Arentoft, T., Kjeldsen, H., Bedding, T. R., et al. 2008, ApJ, 687, 1180

Auvergne, M., Bodin, P., Boisnard, L., et al. 2009, A\&A, 506, 411

Baglin, A., Auvergne, M., Barge, P., et al. 2006, ESA SP, 1306, 33

Barban, C., Deheuvels, S., Baudin, F., et al. 2009, A\&A, 506, 51

Bruntt, H. 2009, A\&A, 506, 235

Carrier, F., De Ridder, J., Baudin, F., et al. 2009, A\&A, accepted

Chaplin, W. J., Appourchaux, T., Arentoft, T., et al. 2008, Astron. Nachr., 329,549

Christensen-Dalsgaard, J., \& Frandsen, S. 1983, Sol. Phys., 82, 469

Christensen-Dalsgaard, J., Arentoft, T., Brown, T. M., et al. 2007, Commun. Asteroseismol., 150, 350

Fischer, R. A. 1935, The Design of Experiments (Edinburgh: Oliver and Boyd), 18
Fossat, E., Kholikov, Sh., Gelly, B., et al. 1999, A\&A, 343, 608

Frohlich, C., Andersen, B. N., Appourchaux, T., et al. 1997, Sol. Phys., 170, 1

Garcia, R. A., Régulo, C., Samadi, R., et al. 2009, A\&A, 506, 41

Kjeldsen, H., Bedding, T. R., Viskum, M., \& Frandsen, S. 1995, AJ, 109, 1313

Kjeldsen, H., et al. 2008, KASC target selection procedure: Instructions,

http://astro.phys.au.dk/KASC/DASC_KASOC_0008_5.pdf

Hekker S., Kallinger, T., Baudin, F., et al. 2009, A\&A, 506, 465

Mathur, S., Garcia, R. A., Regulo, C., et al. 2009, A\&A, submitted [arXiv: 0907.1139]

Max, J., \& Lacoume, J. L. 1996, Méthodes et techniques du traitement du signal (Dunod)

Michel, É., Baglin, A., Auvergne, M., et al. 2008, Science, 322, 558

Mosser, B., Maillard, J. P., Mekarnia, D., \& Gay, J. 1998, A\&A, 340, 457

Mosser, B., Bouchy, F., Catala, C., et al. 2005, A\&A, 431, L13

Mosser, B., Deheuvels, S., Michel, E., et al. 2008, A\&A, 488, 635

Mosser, B., Roxburgh, I., Michel, E., et al. 2009, A\&A, 506, 33

Roxburgh, I. 2009, A\&A, 506, 435

Roxburgh, I. W., \& Vorontsov, S. V. 2006, MNRAS, 369, 1491

Stello, D., Chaplin, W. J., Bruntt, H., et al. 2009, ApJ, 700, 1589

Tassoul, M. 1980, ApJS, 43, 469 